\title{
SECCIÓN TOTAL DEL NERVIO NEUMOGÁSTRICO POSTERIOR A TRAUMA PENETRANTE DE CUELLO: DESCRIPCIÓN DE UN CASO
}

\author{
TOTAL SECTION OF THE VAGUS NERVE AFTER A PENETRATING NECK TRAUMA: A CASE REPORT
} SECÇÃO TOTAL DO NERVO NEUMOGÁSTRICO APÓS TRAUMA PENETRANTE DO PESCOÇO:
DESCRIÇÃO DE UM CASO

\author{
JAMES NEIRA BORJA', COLÓN MANZO VERDEZOTO', BORIS CABALLERO PINEDA², CARLOS LUIZ GUANOLUIZA ${ }^{3}$ \\ ${ }^{1}$ Hospital Luis Vernaza, Guayaquil, Ecuador \\ ${ }^{2}$ Centro de Salud Pueblo Viejo, Los Ríos, Ecuador \\ ${ }^{3}$ Centro de Salud Quinsaloma - IESS, Los Ríos, Ecuador
}

El cuello, cuya área representa aproximadamente el $1 \%$ de la superficie corporal, es una región compleja con múltiples componentes anatómicos, casi todos con importancia vital, que están muy expuestos a diversos tipos de traumatismos como accidentes de tránsito, agresiones, y riesgos deportivos; ante lo cual hay que establecer prioridad de atención médica. Los traumas de cuello pueden ser contusos (o cerrados) causados en su mayoría, por accidentes de tránsito, en los que se deben valorar aspectos como aumento del volumen del cuello, edema o hematomas que pueden progresar y causar disnea, disfagia, enfisema subcutáneo y alteraciones de la voz; este mecanismo ha disminuido con el uso de los cinturones de seguridad. La segunda causa es penetrante 0 abierta, producida por agresiones con armas corto punzante en la cual el daño es limitado, y en menor medida por armas de fuego con consecuencias destructivas y desgarros secundarios. La actuación definitiva debe incluir realización de una cervicotomía lateral amplia con el fin de identificar las lesiones y proceder a repararlas precozmente, más que mantenerlos en observación. En este artículo se presenta el caso de sección total del nervio neumogástrico en un trauma penetrante de cuello con su manejo respectivo.

PALABRAS CLAVE: traumatismos del cuello, traumatismos del nervio craneal, nervio vago.

Abstract

The neck, whose area accounts for about $1 \%$ of the body surface, is a complex region with multiple anatomical components, almost all with vital importance, which are heavily exposed to various kinds of injuries such as traffic accidents, assaults, and sports risks; to which there must be established priority health care. Neck traumas can be blunt (or closed) mostly caused by traffic accidents, in which aspects such as increased neck volume, edema or hematomas must be valued since they may progress and cause dyspnea, dysphagia, subcutaneous emphysema and voice alteration; this occurrence has decreased with the use of seat belts. The second cause is pervasive or open, produced by stabbing attacks with blade weapons in which the damage is limited, and to a lesser extent by firearms with destructive consequences and side tears. The second cause is pervasive or open, produced by stabbing attacks with blade weapons in which the damage is limited, and to a lesser extent by firearms with destructive consequences and side tears. The final performance must include a wide lateral cervicotomy in order to identify lesions and proceed to their early repair rather than monitoring them. The case of total section of the vagus nerve in a penetrating neck trauma and its respective procedure is presented in this article.

KEYWORDS: neck injuries, cranial nerve injuries, vagus nerve.

Resumo

0 pescoço, cuja área representa cerca de $1 \%$ da superfície do corpo, é uma região complexa com múltiplos componentes anatômicos, quase todos com importância vital, que estão muito expostos a vários tipos de lesões como acidentes de trânsito, agressões e riscos desportivos; pelo que se tem que estabelecer a prioridade de atenção médica. Os traumas de pescoço podem ser contusos (ou fechados) causados principalmente por acidentes de trânsito, em que aspectos devem ser avaliados como 0 aumento do volume do pescoço, edema ou hematomas que podem progredir e causar dispneia, disfagia, enfisema subcutâneo e alterações da voz; este mecanismo diminuiu com o uso dos cintos de segurança. A segunda causa é penetrante ou aberta, produzida por ataques com armas perfuro cortantes em que o dano é limitado; e em menor medida pelas armas de fogo com consequências destrutivas e rasgões secundários. A ação definitiva deve incluir a realização de cervicotomia lateral ampla com o objetivo de identificar as lesões e proceder à sua reparação precoce, mais do que manter a observação. Neste artigo apresentamos o caso da secção total do nervo neumogástrico em um trauma penetrante do pescoço com sua gestão respetiva.

PALABRAS-CHAVE: lesões do pescoço, traumatismos dos nervos cranianos, nervo vago. 
INTRODUCCIÓN

Los traumatismos penetrantes del cuello suponen aproximadamente el 2-3\% de la totalidad de las lesiones traumáticas penetrantes, con una mayor incidencia entre los varones $y$, son generalmente, originados por agresiones físicas. ${ }^{1}$ La mayor parte de las lesiones afectan a la vena yugular, la arteria carótida y sus ramas, la vía aérea o digestiva, y en menor escala lesiones neurológicas como el neumogástrico. La mortalidad global de estas lesiones es de un 20 a $40 \%{ }^{1,2}$ La valoración del déficit neurológico es imprescindible, ya que nos aporta información referente a la irrigación cerebral. Los nervios neumogástricos, laríngeo recurrente, espinal accesorio, hipogloso y frénico pueden ser lesionados secundariamente en las heridas del cuello., ${ }^{2,3}$

El nervio vago se origina en la médula oblongada, una parte del tronco encefálico, que está localizado en la parte posterior inferior del cerebro. ${ }^{2-4}$ Es uno de los nervios más largos del cuerpo, ya que va desde el cerebro hasta el colon. Suministra fibras nerviosas a la zona de la garganta, los pulmones, el corazón y los órganos digestivos. Es un nervio mixto: motor, sensitivo y vegetativo, que controla la respuesta inflamatoria. En el trayecto extracraneal puede ocurrir la lesión del nervio recurrente o laríngeo inferior, afectándose más el izquierdo por su recorrido más largo. El neurotransmisor acetilcolina es liberado por el nervio vago para disminuir la respuesta inmunológica antes de que cause daños colaterales. ${ }^{5}$

Los problemas que derivan de las lesiones en cuello son las muertes súbitas o tempranas y las complicaciones, que prolongan la estancia hospitalaria o comprometen la evolución de los lesionados de los compartimentos anterior o lateral de cuello, por lo que deben ser sometidos a exploración quirúrgica tempranamente. ${ }^{4,6,7} \mathrm{~A}$ continuación se presenta el caso de una paciente de 32 años con herida por objeto corto punzante a nivel de cuello y compromiso de estructuras cercanas.

\section{PRESENTACIÓN DEL CASO}

Paciente femenina, 32 años de edad, sin antecedentes patológicos relevantes, es ingresada por familiares al servicio de emergencia del hospital Luis Vernaza por presentar cuadro clínico caracterizado por palidez generalizada, pérdida de continuidad de tejido a nivel cervical antero lateral derecho de la zona topográfica II y III (figura 1) sangrado profuso de una hora de evolución por sufrir herida corto punzante de aproximadamente $15 \mathrm{~cm}$ de longitud, la misma que expone elementos anatómicos vasculonerviosos. Ingresa con signos vitales estables, presión arterial: 110/70, frecuencia cardiaca: 87, FR: 23, saturación de O2: 98 \% y Glasgow: 15/15. Se realizan exámenes de laboratorio que incluyen biometría hemática y tiempos de coagulación con resultados dentro de parámetros normales (tabla 1).

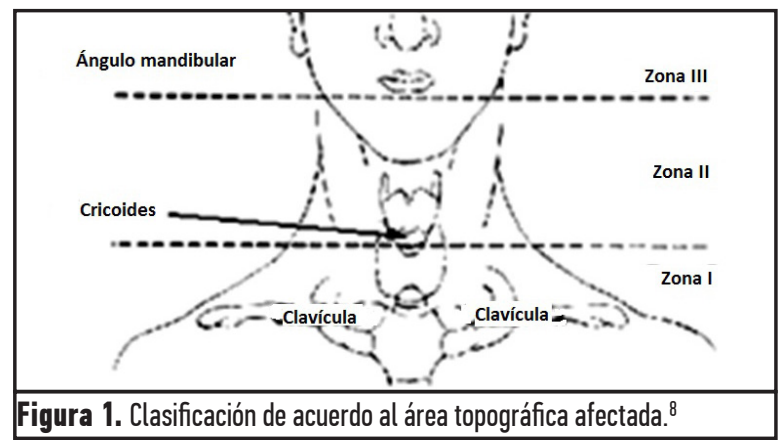

TABLA 1. VALORES DE LABORATORIO EN EL SERVICIO DE EMERGENCIA

\begin{tabular}{lc} 
EXÁMENES DE LABORATORIO & \\
\hline Leucocitos & $6.23 \mathrm{~K} / \mathrm{uL}$ \\
\hline Neutrófilos & $5.60 \mathrm{~K} / \mathrm{uL}$ \\
\hline Linfocitos & $8.5 \mathrm{~K} / \mathrm{uL}$ \\
\hline Monocitos & $1.6 \mathrm{~K} / \mathrm{uL}$ \\
\hline Eosinófilos & $0.0 \mathrm{~K} / \mathrm{uL}$ \\
\hline Hemoglobina & $12.1 \mathrm{~g} / \mathrm{dL}$ \\
\hline Hematocrito & $37.1 \%$ \\
\hline Plaquetas & $137 \mathrm{~K} / \mathrm{uL}$ \\
\hline TP & $11.1 \mathrm{seg}$. \\
TPT & $25.9 \mathrm{seg}$. \\
\hline
\end{tabular}

La paciente es ingresada con diagnóstico preoperatorio de trauma vasculonervioso de cuello. El servicio de Cirugía General decide la exploración quirúrgica inmediata, se realiza cervicotomía lateral amplia debido a que herida atravesó el músculocutáneo del cuello, se efectuó exploración quirúrgica siguiendo los criterios de Ronn y Christensen (tabla 2) (figura 2), ${ }^{1,3}$

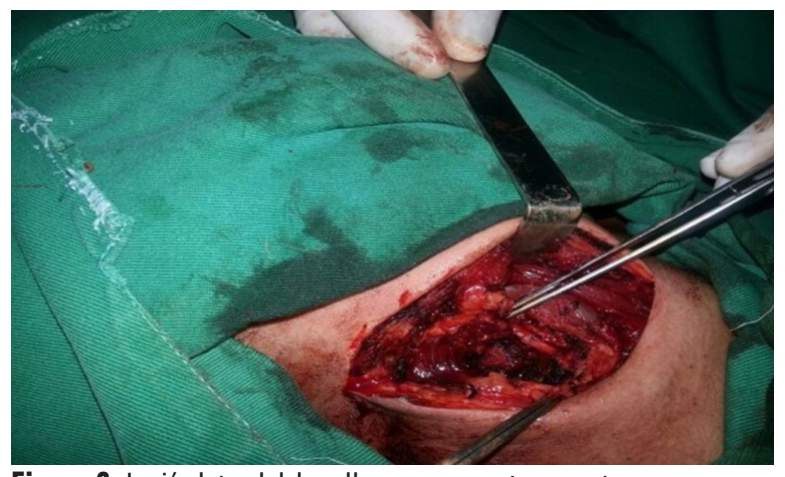

Figura 2. Lesión lateral del cuello por arma cortopunzante. 
TABLA 2. CRITERIOS DE INTERVENCIÓN QUIRÚRGICA DE ROON Y CHRISTENSEN ${ }^{8}$

\begin{tabular}{|c|c|c|}
\hline GRADO & PROFUNDIDAD DE LA LESIÓN & REQUERIMIENTO \\
\hline Grado & $\begin{array}{l}\text { Herida penetrante del cuello que no } \\
\text { sobrepasa la plastina }\end{array}$ & No cirugía \\
\hline $\begin{array}{l}\text { Grado } \\
\|\end{array}$ & $\begin{array}{l}\text { Herida penetrante del cuello que sobrepasa } \\
\text { la plastina sin signos y síntomas de lesión } \\
\text { vascular o de la vía aérea }\end{array}$ & $\begin{array}{l}\text { Cirugía después } \\
\text { de evaluación } \\
\text { secundaria }\end{array}$ \\
\hline $\begin{array}{l}\text { Grado } \\
\text { III }\end{array}$ & $\begin{array}{l}\text { Herida penetrante del cuello con lesión } \\
\text { vascular o de la vía aérea con o sin signos } \\
\text { neurológicos }\end{array}$ & Cirugía inmediata \\
\hline $\begin{array}{l}\text { Grado } \\
\text { IV }\end{array}$ & $\begin{array}{l}\text { Herida penetrante del cuello con hemorragia } \\
0 \text { asfixia inminente }\end{array}$ & $\begin{array}{l}\text { No requiere } \\
\text { fallece }\end{array}$ \\
\hline
\end{tabular}

Se evidencia del lado derecho sección total de músculos escalenos anterior y posterior, sección parcial de fascículo clavicular de esternocleidomastoideo, sección total del nervio neumogástrico a nivel de C4 (figura 3), sección total del nervio cervical superficial, y sección total de vena yugular externa.

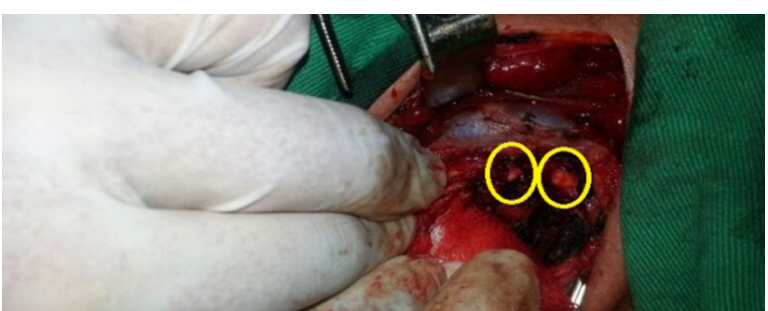

Figura 3. Sección total del Nervio Neumogástrico Derecho (círculos amarillos).

Se procede a realizar una exploración vascular de cuello más ligadura de yugular externa derecha seccionada y rafia de los nervios neumogástrico y cervical superficial derecho totalmente seccionados (figura 4).

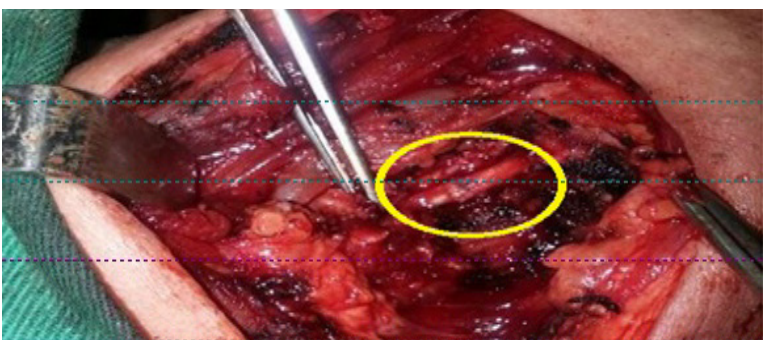

Figura 4. Anastomosis término terminal del Nervio Neumogástrico derecho con Prolene 6/0.

La lesión fue reparada con prolene $6 / 0$, no se colocaron drenes y se procedió a cerrar por planos. Ingresó a post-operatorio y a las 24 horas pasó a sala de cirugía general. Cuarenta y ocho horas después toleró vía oral, no presentó ningún tipo de secuelas neurológicas, funcionales, ni infecciosa dándose de alta a los cinco días del procedimiento quirúrgico.
Se realizó electromiografía (EMG) encontrándose dentro de parámetros normales y se refiere al servicio de Medicina Física y Rehabilitación para continuar con su recuperación.

\section{DISCUSIÓN}

Al momento del ingreso no se pudo observar ninguna complicación sistémica provocada por sección total del neumogástrico, la paciente no manifestaba síntomas o signos de lesión neurológica, y por la gravedad de la lesión anatómica sufrida era muy difícil identificar complicación alguna. La valoración del déficit neurológico es imprescindible, ya que nos aporta información referente a la irrigación cerebral. Los pacientes inestables, sin importar el nivel de compromiso, se llevan al quirófano inmediatamente. Los pacientes con trauma de cuello deben ser sometidos a exploración quirúrgica, de acuerdo a los Criterios de intervención quirúrgica de Roon y Christensen: Toda herida que atraviesa el músculo cutáneo del cuello debe someterse a exploración quirúrgica aun cuando el paciente no manifieste síntomas o signos de lesión. ${ }^{8}$

La tasa de éxito después de una reparación del neumogástrico, como de cualquier otra estructura anatómica del cuello, es cerca del $100 \%$ cuando se la realiza con la técnica adecuada. Un fracaso en la misma conlleva a secuelas definitivas de tipo irreversible. Por eso es de vital importancia para un cirujano conocer la región anatómica del cuello y las técnicas quirúrgicas adecuadas. ${ }^{9}$

Dado que el nervio vago inerva la garganta, un daño en él suele conllevar problemas para hablar y deglutir. ${ }^{10,11}$ A menudo también se produce una pérdida del reflejo nauseoso. Este nervio envía fibras nerviosas al estómago, por lo que una lesión en él puede causar problemas digestivos como gastroparesia, provocando sensación de plenitud tras comer una pequeña cantidad de alimentos, la pérdida de peso, el ardor de estómago y las náuseas. ${ }^{6,9,12,13}$ Puede darse también lesión en el nervio laríngeo recurrente provocando parálisis de cuerdas vocales, disfonía y voz ronca. Otra causa de daño en el nervio vago es que se produzca un traumatismo en él, esto puede estar causado por un golpe recibido por el cuerpo (a consecuencia de un accidente de automóvil) que ejerce una presión en parte del nervio vago. Las remisiones espontáneas son comunes en la neuralgia del vago asociada a la del glosofaríngeo. No hay que olvidar que el vago izquierdo tiene más probabilidad de lesionarse que el derecho 
debido a su trayecto más largo (cinco veces más frecuente que el lado derecho). Es difícil saber con exactitud el porcentaje de cada tipo de causa, pero por traumatismo cervical es del $1 \%{ }^{13,14}$

El concepto que debe quedar es que el vago es el nervio que más tareas realiza. De tal manera que para descartar alguna complicación posterior a la anastomosis se realizó en la paciente una electromiografía (EMG) arrojando valores negativos para una posible hiperestesia pericicatrizal; de todas formas se sugirió que acuda al servicio de Medicina Física y Rehabilitación para su manejo.

De acuerdo a la revisión de la literatura universal no se pudo encontrar algún trabajo que reporte sección total del nervio neumogástrico sin lesión vascular interna. Por este motivo se comparte este caso de lesión nerviosa sin compromiso vascular secundario a trauma de cuello junto a las circunstancias que consiguieron un resultado favorable para el paciente.

CONCLUSIONES

Las lesiones penetrantes en la región cervical son en su mayoría resultado de agresiones por armas de fuego, accidentes automovilísticos o armas punzocortantes. ${ }^{15,16}$ El cuello es donde se ubican numerosas estructuras y órganos vitales. Las alteraciones mayormente encontradas en estos pacientes son lesión de estructuras vasculares, nerviosas, faringoesofágicas y laringotraqueales; siendo excepcionales las lesiones penetrantes que no comprometen ninguna de estas estructuras. ${ }^{17}$ Resulta muy importante realizar la exploración quirúrgica aunque no existan signos de inestabilidad para conseguir un tratamiento precoz en caso de ser necesario y así evitar secuelas funcionales a largo plazo. ${ }^{18}$

\section{REFERENCIAS BIBLIOGRÁFICAS}

1. Flores J, Ortiz de la Peña J, Cervantes J. Trauma penetrante del cuello: ¿Es confiable la exploración física para el diagnóstico de lesiones? An Méd. 2000;45(1):6-12.

2. Demetriades D, Asensio JA, Velmahos G, Thal E. Complex problems in penetrating neck trauma. Surg Clin North Am. agosto de 1996;76(4):661-83.

3. Garantziotis S, Kyrmizakis DE, Liolios AD. Critical care of the head and neck patient. Crit Care Clin. enero de 2003; 19 (1): 73-90.

4. Pierre EJ, McNeer RR, Shamir MY. Early management of the traumatized airway. Anesthesiol Clin. marzo de 2007;25(1):1-11, vii.
5. Rosas-Ballina M, Olofsson PS, Ochani M, Valdés-Ferrer SI, Levine YA, Reardon C, et al. Acetylcholine-synthesizing T cells relay neural signals in a vagus nerve circuit. Science. el 7 de octubre de 2011;334(6052):98-101.

6. Rathlev NK, Medzon R, Bracken ME. Evaluation and management of neck trauma. Emerg Med Clin North Am. agosto de 2007;25(3):679-94, viii.

7. Reyes López D, Ibarra Guillén JA, Cabrera Rayo A, Morales Salas R, Sánchez Pompa M, Laguna Hernández G, et al. Lesión penetrante por objeto punzocortante en cuello. Reporte de un caso y revisión de la literatura. Arch Med Urgenc México. 2009;1(2):63-6.

8. Headache Classification Subcommittee of the International Headache Society. The International Classification of Headache Disorders: 2nd edition. Cephalalgia Int J Headache. 2004;2 4 Suppl 1:9-160.

9. Asensio JA, Valenziano CP, Falcone RE, Grosh JD. Management of penetrating neck injuries. The controversy surrounding zone II injuries. Surg Clin North Am. abril de 1991;71(2):267-96.

10. Wood J, Fabian TC, Mangiante EC. Penetrating neck injuries: recommendations for selective management. J Trauma. mayo de 1989; 29(5): 602-5.

11. Golueke PJ, Goldstein AS, Sclafani SJ, Mitchell WG, Shaftan GW. Routine versus selective exploration of penetrating neck injuries: a randomized prospective study. J Trauma. diciembre de 1984; 24(12): 1010-4.

12. Bagheri SC, Khan HA, Bell RB. Penetrating Neck Injuries. Oral Maxillofac Surg Clin N Am. agosto de 2008; 20(3): 3 93-414.

13. Herrera FA, Mareno JA, Easter D. Management of Penetrating Neck Injuries: Zone II. J Surg Educ. el 1 de marzo de 2007; 64(2): 75-8.

14. Demetriades D, Theodorou D, Cornwell E, Weaver F, Yellin A, Velmahos G, et al. Penetrating injuries of the neck in patients in stable condition. Physical examination, angiography, or color flow Doppler imaging. Arch Surg Chic Ill 1960. septiembre de 1995; 130(9): 971-5.

15. Rontal M, Rontal E. Lesions of the vagus nerve: diagnosis, treatment and rehabilitation. The Laryngoscope. enero de 1977; 87(1): 72-86.

16. Perello J. Neuralgia of the superior laryngeal nerve caused by microsurgery. An Otorrinolaringológicos Ibero-Am. 1974; 1(3): 73 .

17. Taha JM, Tew JM. Long-term results of surgical treatment of idiopathic neuralgias of the glossopharyngeal and vagal nerves. Neurosurgery. mayo de 1995; 36(5): 926-30; discussion 930-1.

18. Rushton JG, Stevens JC, Miller RH. Glossopharyngeal (vagoglossopharyngeal) neuralgia: a study of 217 cases. Arch Neurol. abril de 1981; 38( 4): 201-5. 